\title{
An accurate numerical scheme for the contraction of a bubble in a Hele-Shaw cell
}

\author{
Michael C. Dallaston ${ }^{1} \quad$ Scott W. $\mathrm{McCue}^{2}$
}

(Received 18 October 2012; revised 1 June 2013)

\begin{abstract}
We report on an accurate numerical scheme for the evolution of an inviscid bubble in radial Hele-Shaw flow, where the nonlinear boundary effects of surface tension and kinetic undercooling are included on the bubble-fluid interface. As well as demonstrating the onset of the Saffman-Taylor instability for growing bubbles, the numerical method is used to show the effect of the boundary conditions on the separation (pinch off) of a contracting bubble into multiple bubbles, and the existence of multiple possible asymptotic bubble shapes in the extinction limit. The numerical scheme also allows for the accurate computation of bubbles which pinch off very close to the theoretical extinction time, raising the possibility of computing solutions for the evolution of bubbles with non-generic extinction behaviour.
\end{abstract}

http://journal. austms.org.au/ojs/index.php/ANZIAMJ/article/view/6241 gives this article, (c) Austral. Mathematical Soc. 2013. Published June 9, 2013, as part of the Proceedings of the 16th Biennial Computational Techniques and Applications Conference. ISSN 1446-8735. (Print two pages per sheet of paper.) Copies of this article must not be made otherwise available on the internet; instead link directly to this URL for this article. 
Subject class: 76D27, 76E17, 65E05

Keywords: Hele-Shaw flow, bubble contraction, Saffman-Taylor instability

\section{Contents}

1 Introduction

C310

1.1 Formulation . . . . . . . . . . . . . . . .

C311

1.2 Expanding bubbles . . . . . . . . . . . . . . . . C C312

1.3 Contracting bubbles . . . . . . . . . . . . C315

2 Numerical scheme and results

C315

2.1 Unscaled method . . . . . . . . . . . . . . . .

C316

2.2 Scaled method

2.3 Results .

3 Discussion

C323

References

C323

\section{Introduction}

In applied mathematics, expanding or contracting bubbles in a Hele-Shaw cell is a canonical example of a free boundary problem, where, along with Laplace's equation, one must solve for the position of a moving interface. It serves both mathematically and experimentally as a model for more complicated free boundary problems, for instance Stefan (melting/freezing) problems [16, 17], and groundwater flow [19]. Expanding bubbles are frequently a topic of numerical methods [8]; in this case the bubble exhibits the Saffman-Taylor instability [19], with the interface developing long fingering patterns, which presents a significant challenge for numerical schemes. 
The case of contracting bubbles is less studied, although there are interesting phenomena involved that are still active topics of research. A contracting bubble may shrink to a single point $[6,14]$ or, depending on the initial shape, separate or 'pinch off' into multiple bubbles before the time of extinction [5, 11]. We describe an accurate and efficient numerical scheme that focuses on contracting bubbles (although it is still applicable to expanding ones), including a rescaled version that allows for the shape of a single bubble to be accurately computed at a point very close to its extinction point. Our numerical scheme allows for the inclusion of the nonlinear boundary effects of surface tension and kinetic undercooling.

In the remainder of this section we describe the problem and derive the nearcircle stability analysis, which indicates the effect of the nonlinear boundary conditions on expanding or contracting bubbles. In Section 2, we describe our numerical scheme and use it to demonstrate phenomena for contracting bubbles: the effect of surface tension and kinetic undercooling on pinch off, and the accurate computation of a bubble which pinches off very close to its theoretical extinction time, which raises the possibility of non-generic asymptotic bubble shapes [10]. Lastly, we demonstrate the existence of multiple asymptotic extinction shapes for certain parameter values, which we recently reported on elsewhere [2]. We discuss possible directions of future study in Section 3.

\subsection{Formulation}

The equations describing the two dimensional Hele-Shaw problem with surface tension and kinetic undercooling are well known $[9,18]$. We consider a single connected inviscid bubble $\Omega(\mathrm{t}) \subset \mathbb{R}^{2}$, with surface tension and kinetic undercooling acting on the boundary $\partial \Omega(t)$, where the area $\mathcal{A}$ of the bubble is changing at a constant rate:

$$
\frac{\mathrm{d} \mathcal{A}}{\mathrm{dt}}=-2 \pi \mathrm{Q} .
$$


Let $\phi$ be the velocity potential of the viscous fluid outside the bubble. The potential is related to the fluid pressure $p$ by $\phi=-k\left(p-p_{0}\right)$, where $p_{0}$ is the pressure in the bubble (assumed constant) and $k$ is a constant dependent on the fluid viscosity and plate separation width. The free boundary problem for $\phi$ is

$$
\begin{aligned}
\nabla^{2} \phi & =0 \quad(x, y) \in \mathbb{R}^{2} \backslash \Omega(t), \\
v_{n} & =\frac{\partial \phi}{\partial n} \quad(x, y) \in \partial \Omega(t), \\
\phi & =c v_{n}+\sigma \kappa \quad(x, y) \in \partial \Omega(t), \\
\phi & \sim-Q \log \sqrt{x^{2}+y^{2}} \quad(x, y) \rightarrow \infty,
\end{aligned}
$$

where $v_{n}$ is the normal velocity of the interface, and $c$ and $\sigma$ are the kinetic undercooling and surface tension parameters, respectively. Contracting bubbles correspond to $\mathrm{Q}>0$, while expanding bubbles have $\mathrm{Q}<0$ (see Figure 1). While we consider a constant source term for simplicity, the methods of this article extend readily to a time dependent source term $Q=Q(t)$, which is a topic of current interest in the area of controlling the viscous instability $[4,3,13]$.

\subsection{Expanding bubbles}

In the case of expanding bubbles $(\mathrm{Q}<0)$ the interface exhibits the SaffmanTaylor instability [19], and develops long 'fingers' of the inviscid fluid. In the absence of regularisations such as surface tension, the interface generically develops cusps (curvature singularities) in finite time [9, 1], while surface tension suppresses these singularities, allowing the solution to continue for all time; the interface develops long fingers that leave behind 'fjords' of viscous fluid [12]. Surface tension in this model serves the same purpose as finite viscosity in ill-posed ideal flow problems exhibiting Rayleigh-Taylor or KelvinHelmholtz instabilities, which otherwise develop curvature singularities [7, e.g.]. 


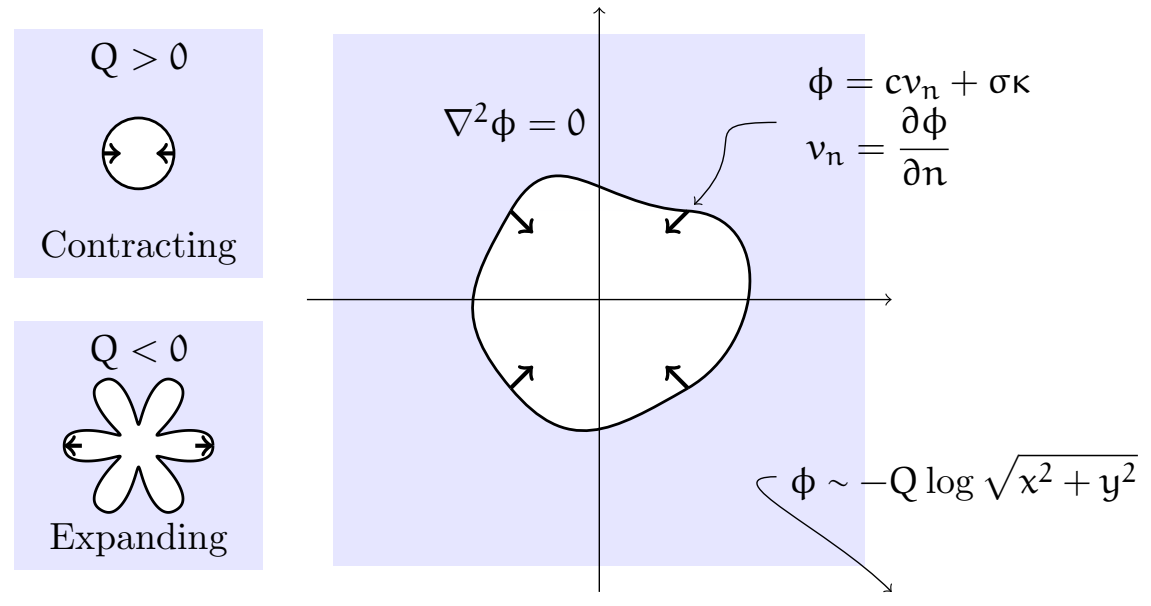

Figure 1: A schematic of the problem (2)-(5) of a bubble in a Hele-Shaw cell with surface tension and kinetic undercooling effects considered on the boundary.

The suppression of singularities is evident in the following linear stability analysis, as surface tension stabilises the highest order modes. The system (2)(5) admits as a solution the perfectly circular bubble of radius $s_{0}(t)$ where

$$
\frac{\mathrm{ds} \mathrm{s}_{0}}{\mathrm{dt}}=-\frac{\mathrm{Q}}{\mathrm{s}_{0}} .
$$

Linear stability analysis [2] shows that if the circle is perturbed by an $n$th mode term, that is the interface is given by $s(\theta, t)=s_{0}+\epsilon \gamma_{n} \cos n \theta$ in polar coordinates, then the amplitude $\gamma_{\mathrm{n}}$ of this perturbation evolves according to

$$
\frac{1}{\gamma_{n}} \frac{d \gamma_{n}}{d s_{0}}=\frac{n-1}{s_{0}+n c}+\frac{\sigma}{Q} \frac{n\left(n^{2}-1\right)}{s_{0}\left(s_{0}+n c\right)} .
$$

For expanding bubbles $(\mathrm{Q}<0)$ it follows that for $\sigma>0$ and $c \geqslant 0$, the high modes $\gamma_{n}$ decay for all sufficiently large $n$, whereas arbitrarily high modes are unstable for $\sigma=0$. Thus surface tension suppresses curvature singularities that occur in the zero surface tension case. Additionally, the second mode $\gamma_{2}$ 
(a)

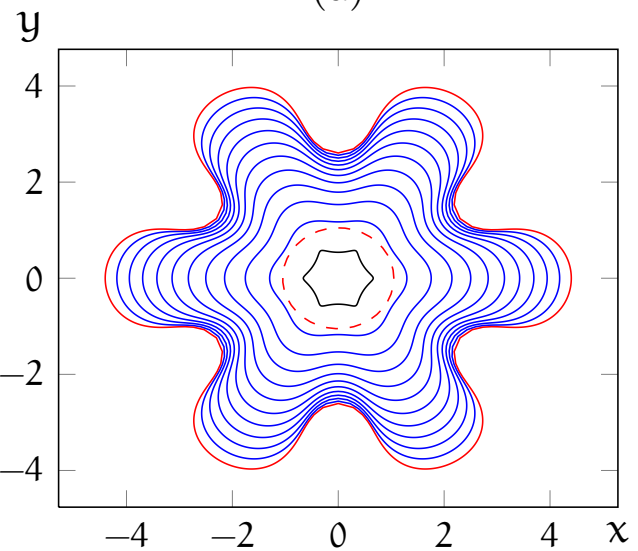

(b)

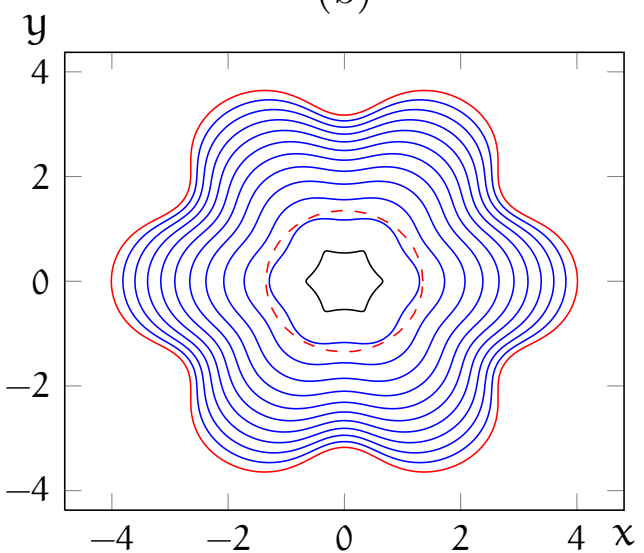

Figure 2: The evolution of an expanding bubble with six-fold symmetry, with flux $Q=-1$, surface tension $\sigma=0.02$, and kinetic undercooling (a) $c=0$ and $(b) c=0.2$ (initial and final interfaces are black and red, respectively). The radius beyond which the sixth mode perturbation becomes unstable (found from (8)) is shown in dashed red. Kinetic undercooling acts to increase this radius and delay the formation of fingers.

is always stable, while the $n$th mode is stable when the radius $s_{0}$ is sufficiently small, so that it satisfies

$$
s_{0}<\frac{n}{n-2}\left[c+\frac{\sigma}{(-Q)}\left(n^{2}-1\right)\right], \quad n \geqslant 3 .
$$

Increasing surface tension $\sigma$ or kinetic undercooling $c$ in (8) increases the radius at which the $n$th mode becomes unstable. In Figure 2 we demonstrate this onset of instability of the sixth mode of perturbation, first with surface tension alone, and then with both surface tension and kinetic undercooling. These solutions were computed using the numerical scheme described in Section 2. 


\subsection{Contracting bubbles}

The case of contracting bubbles corresponds to $t$ increasing and $Q>0$ in (2)(5). For zero surface tension and zero kinetic undercooling, bubbles generically shrink to ellipses unless they pinch off first $[6,5,15,10]$. For nonzero surface tension the extinction shape is generically circular, although pinch off is still a possibility. When both nonlinear boundary effects are included (7) implies they are in competition, with surface tension stabilising the boundary and kinetic undercooling acting to destabilise it. We use the numerical scheme of the next section to demonstrate the phenomenon of pinch off with these nonlinear boundary effects. We find that surface tension acts to delay or prevent pinch off, whereas kinetic undercooling causes it to occur earlier than otherwise.

If the bubble does not pinch off but instead shrinks to a single point, then there is a complex bifurcation structure in the asymptotic bubble shape, which we recently reported [2]. We omit the details here; however, we numerically demonstrate the existence of multiple extinction shapes for certain parameter values in the next section (see Figure 5).

\section{$2 \quad$ Numerical scheme and results}

In this section we describe our numerical scheme for solving the system (2)-(5). This scheme is based on a spectral (power series) representation of a (complexvalued, analytic) conformal mapping function from the unit disc to the evolving fluid region. We also describe a scaled version of the method that allows the evolution of a bubble very close to extinction to be accurately captured. 


\section{$2.1 \quad$ Unscaled method}

Let $z=x+$ iy be the complex spatial variable, and $z=g(\zeta, t)$ be a timedependent mapping function which maps the unit disc in the $\zeta$ plane conformally to the fluid region $\Omega(t)$. The mapping function $g$ is analytic in the punctured unit disc, with a simple pole at the origin corresponding to the far-field in the physical plane. The mapping function is determined by the conditions on the interface, which may be written entirely in terms of $\mathrm{g}$ on the unit circle:

$$
\mathfrak{R}\left\{\mathrm{g}_{\mathrm{t}} \overline{\bar{\zeta} \mathbf{g}_{\zeta}}\right\}=\mathfrak{R}\left(\zeta \Phi_{\zeta}\right)=\mathrm{Q}-\operatorname{c} \mathfrak{R}\left\{\zeta \mathrm{V}_{\zeta}\right\}-\sigma \mathfrak{R}\left\{\zeta \mathrm{K}_{\zeta}\right\}, \quad|\zeta|=1,
$$

where $\Phi=\phi+i \mathcal{i}$ is the complex velocity potential, and $\mathrm{V}$ and $\mathrm{K}$ are complex analytic functions in the unit disc whose real boundary data are equal to the normal velocity and curvature respectively:

$$
\mathfrak{R}\{\mathrm{V}\}=v_{\mathfrak{n}}=\frac{\mathfrak{R}\left\{\mathbf{g}_{\mathrm{t}} \overline{\zeta \mathbf{g}_{\zeta}}\right\}}{\left|\boldsymbol{\zeta g}_{\zeta}\right|}, \quad \mathfrak{R}\{\mathrm{K}\}=\kappa=\frac{\mathfrak{R}\left\{\zeta\left(\zeta \mathrm{g}_{\zeta}\right)_{\zeta} \overline{\zeta \mathbf{g}_{\zeta}}\right\}}{\left|\zeta \mathbf{g}_{\zeta}\right|^{3}},
$$

on $|\zeta|=1$. For simplicity and numerical stability we assume symmetry in the $x$ and $y$ axes. The mapping function $g$, along with its spatial and time derivatives, has the power series representation

$$
\begin{aligned}
& g(\zeta, t)=\sum_{n=0}^{\infty} a_{n}(t) \zeta^{2 n-1}, \quad \zeta g_{\zeta}=\sum_{n=0}^{\infty}(2 n-1) a_{n}(t) \zeta^{2 n-1}, \\
& \zeta\left(\zeta g_{\zeta}\right)_{\zeta}=\sum_{n=0}^{\infty}(2 n-1)^{2} a_{n}(t) \zeta^{2 n-1}, \quad g_{t}=\sum_{n=0}^{\infty} \dot{a}_{n}(t) \zeta^{2 n-1},
\end{aligned}
$$

where $a_{n}(t)$ are unknown coefficients; symmetry in the $x$ axis implies that the $a_{n}$ are real.

To solve for the evolution of the coefficients $a_{n}(t)$ we truncate the series at $\mathrm{N}$ terms. The $\mathrm{N}$ fully implicit equations that we require come from satisfying (9) at $\mathbf{N}$ equally spaced points

$$
\zeta_{j}=e^{i \pi j / 4 N}, \quad j=0, \ldots, N-1,
$$


which lie in the first quadrant on the unit circle. To compute these implicit equations we first evaluate $\mathrm{g}$, its derivatives, and subsequently $\nu_{\mathrm{n}}$ and $\kappa$, at each of the $\zeta_{j}$. Computing $V$ and $K$ from $\nu_{n}$ and $K$ is very simple in the space of their power series coefficients; for example,

$$
v_{n}=\sum_{n=-\infty}^{\infty} b_{n} \zeta^{n} \Rightarrow v=b_{0}+\sum_{n=1}^{\infty} 2 b_{n} \zeta^{n},
$$

plus an arbitrary imaginary constant we take to be zero (note that $b_{\mathfrak{n}}=\bar{b}_{\mathfrak{n}}$, since $v_{n}$ is real). From here we evaluate $\zeta \mathrm{V}_{\zeta}$ and $\zeta \mathrm{K}_{\zeta}$ by differentiation of the power series and substitute into (9). The coefficients $\boldsymbol{a}_{\mathfrak{n}}$ must be advanced in time using a fully implicit ODE solver; we implement our method in Matlab and use the implicit time stepping algorithm ode15i.

\subsection{Scaled method}

To examine the behaviour of bubbles very close to extinction the above method is not optimal. Instead, we carry out a time dependent rescaling in space, which means the bubble is always $\mathcal{O}(1)$ in size. This rescaling requires the assumption that the bubble remains connected. Let $\lambda(t)$ be a characteristic length scale of the bubble that goes to zero as the bubble approaches extinction, and define a new mapping function $\mathrm{G}$ and time like variable $\mathrm{T}$ :

$$
g(\zeta, t)=\lambda G(\zeta, T), \quad T=-\log \lambda .
$$

The image of the unit circle under $G$ is now the bubble shape scaled to be of size $\mathcal{O}(1)$, and time is stretched such that as the bubble tends to extinction $\lambda \rightarrow 0^{+}$and therefore $T \rightarrow \infty$. We again write $G$ as a power series:

$$
\mathrm{G}(\zeta, T)=\sum_{n=-1}^{\infty} A_{n}(T) \zeta^{n}
$$


The area $\mathcal{A}$ of the bubble is

$$
\mathcal{A}=\frac{1}{2 i} \oint_{\partial \Omega} \bar{z} \mathrm{~d} z=-\frac{\lambda^{2}}{2 i} \oint_{|\zeta|=1} \mathrm{G}(1 / \zeta, \mathrm{T}) \mathrm{G}_{\zeta}(\zeta, \mathrm{T}) \mathrm{d} \zeta=-\pi \lambda^{2} \sum_{n=0}^{\infty} n A_{n}^{2}
$$

(the latter a consequence of the residue theorem). Differentiating with respect to unscaled time $t$ and using (1) we obtain

$$
\dot{\lambda} \lambda=\frac{1}{\mathrm{QB}(\mathrm{T})}, \quad \mathrm{B}(\mathrm{T})=\sum_{n=0}^{\infty} n \mathrm{~A}_{\mathrm{n}}\left(\mathrm{A}_{\mathrm{n}}-\mathrm{A}_{\mathrm{n}}^{\prime}\right)
$$

(here the prime ' represents differentiation with respect to T). The governing equation (9) becomes

$$
e^{-\mathrm{T}}\left[\mathfrak{R}\left\{\left(\mathrm{G}-\mathrm{G}_{\mathrm{T}}\right) \overline{\zeta \mathrm{G}_{\zeta}}\right\}+\mathrm{QB}\right]=\mathrm{QBc} \mathfrak{R}\left\{\zeta \widehat{\nabla}_{\zeta}\right\}+\sigma \mathfrak{R}\left\{\zeta \widehat{\mathrm{R}}_{\zeta}\right\}, \quad|\zeta|=1,
$$

where $\widehat{V}$ and $\widehat{R}$ are the functions analytic in the unit disc such that

$$
\mathfrak{R}\{\widehat{\nabla}\}=\frac{\mathfrak{R}\left\{\left(\mathrm{G}-\mathrm{G}_{\mathrm{T}}\right) \overline{\zeta \mathrm{G}_{\zeta}}\right\}}{\left|\zeta \mathrm{G}_{\zeta}\right|}, \quad \mathfrak{R}\{\hat{\mathrm{K}}\}=\frac{\mathfrak{R}\left\{\zeta\left(\zeta \mathrm{G}_{\zeta}\right)_{\zeta} \overline{\zeta \mathrm{G}_{\zeta}}\right\}}{\left|\zeta \mathrm{G}_{\zeta}\right|^{3}}
$$

on $|\zeta|=1$.

We have not yet specified the scaling $\lambda$. Given our assumptions of symmetry, the bubble will always be centred about the origin which will therefore be the extinction point, assuming pinch off does not occur. Subsequently, we use the positive $x$ intercept $\lambda(t)=g(1, t)$ as the scaling, noting that $\lambda \rightarrow 0^{+}$ as the bubble shrinks to a point, as required. This is equivalent to setting

$$
\mathrm{G}(1, \mathrm{~T})=1
$$

To fix the scaling parameter $\lambda$ we replace one of the equations from the discretisation of (17) with (19). Otherwise, the coefficients $A_{n}$ are evolved in the same manner as the $a_{n}$ in the unscaled version. 
Both the scaled and unscaled versions are very efficient as we use the fast Fourier transform to compute boundary values from power series representations, and vice-versa. In producing the numerical results we used $\mathrm{N}=128$ terms. The scheme runs in the order of seconds on a modern desktop computer. The spatial discretisation converges spectrally in number of modes $\mathrm{N}$, thus the major source of numerical error for contracting bubbles is generally in the time stepping. However, for expanding bubbles, the formation of fingers (corresponding to singularities near the unit disc in the $\zeta$ plane) has a strong negative impact on the convergence of the power series (11). This method is thus most effective for contracting bubbles, while not well suited to resolving expanding bubbles far into the regime of finger formation.

\section{$2.3 \quad$ Results}

In addition to the solutions for expanding bubbles shown in Figure 2, computed using the unscaled method above, we examine both the phenomenon of pinch off and the existence of multiple asymptotic bubble shapes, which both require the scaled method to treat the problem near the extinction time.

To examine pinch off we use an initially 'peanut' shaped bubble of the form

$$
\mathrm{G}(\zeta, 0)=\frac{\zeta^{-1}+\alpha \sin \zeta}{1+\alpha \sin 1}, \quad 0<\alpha<1,
$$

where the parameter $\alpha$ controls the initial shape of the bubble; the larger $\alpha$, the closer to pinch off (the denominator in (20) ensures that (19) is satisfied at the initial time). In Figure 3 we plot the numerical solutions of a bubble with this peanut shaped initial condition, using our scaled method. This plot demonstrates the impact of the two nonlinear boundary conditions: surface tension acts to delay or even prevent pinch off, while kinetic undercooling induces pinch off, or causes it to occur at an earlier time than when kinetic undercooling is zero. 
(a)

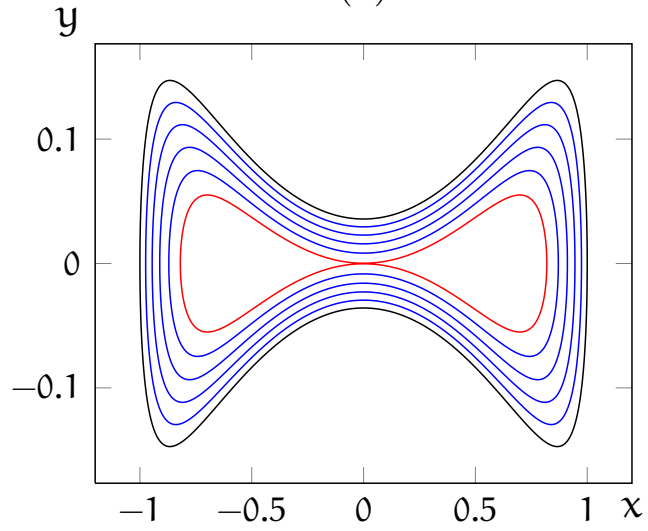

(b)

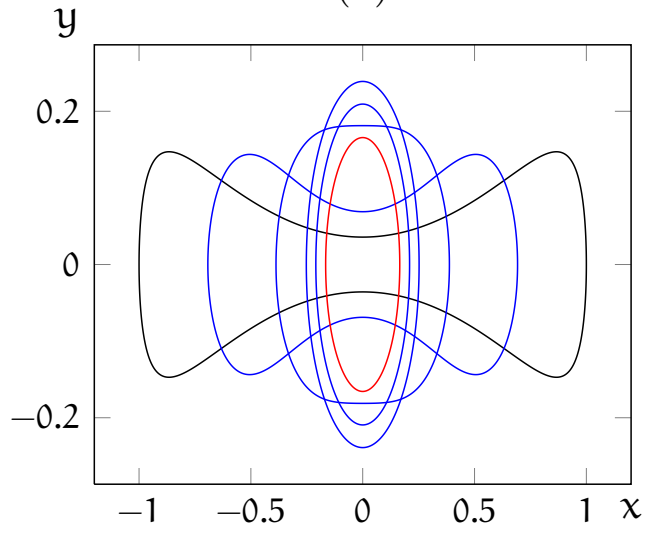

(c)

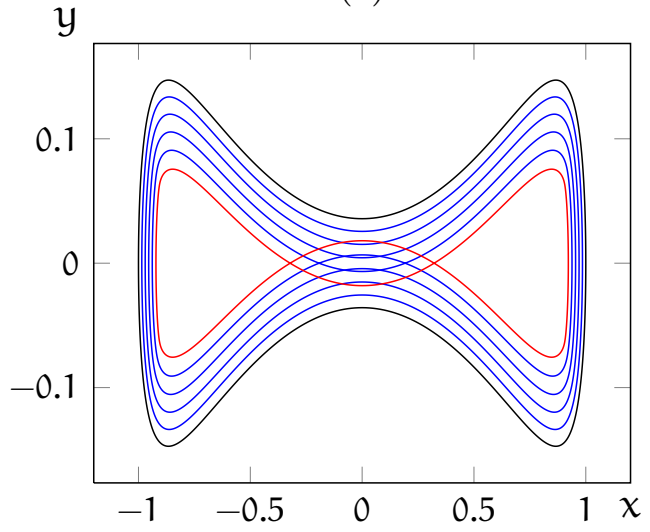

Figure 3: Three examples of the evolution of an initially peanut shaped bubble (20) (with $\alpha=0.8$ ) with $Q=1$ and over the same time period $t \in[0,0.041]$ (initial and final interfaces are black and red, respectively): (a) $\sigma=\mathrm{c}=0$, and the bubble pinches off in finite time; (b) $\sigma=1, \mathrm{c}=0$, and the surface tension prevents pinch off; (c) $\sigma=0, c=1$, and the kinetic undercooling causes pinch off to occur at an earlier time, resulting in an unphysical self-overlapping of the boundary. 


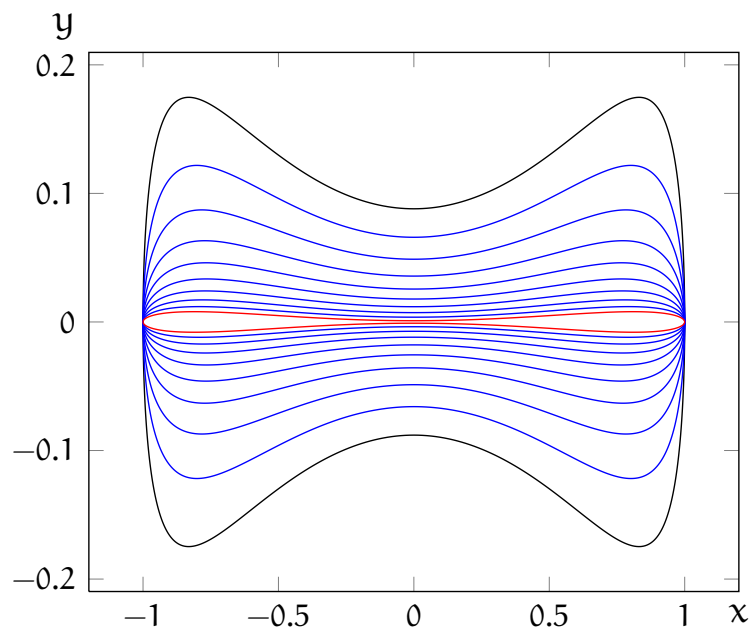

Figure 4: The evolution of initially peanut shaped bubble (20) (with $\alpha=0.72$ ) using the scaled scheme outlined in Section 2 with $Q=1$ and $\sigma=c=0$ (initial and final interfaces are black and red, respectively). The bubble pinches off when the bubble has nearly contracted to a point.

Another interesting topic is what happens in the borderline case between pinch off and extinction. For an initial condition given by (20) with $\alpha$ sufficiently small there is no pinch off and for $\sigma=\mathrm{c}=0$ the asymptotic bubble shape is elliptic. For sufficiently large $\alpha$ pinch off occurs. There must be a borderline value of $\alpha$ between the two regimes; in this borderline case the limiting shape of the bubble is not elliptical even in the absence of surface tension and kinetic undercooling (this special case is discussed by King and McCue [10]). Our scaled method is particularly effective in the numerical solution of bubbles near this borderline case; in Figure 4 we plot the evolution of a bubble that pinches off when the bubble has nearly contracted to a point.

Finally, we use our scheme to show the existence of multiple asymptotic (extinction-limit) bubble shapes. We choose an initially elliptical initial 
(a)

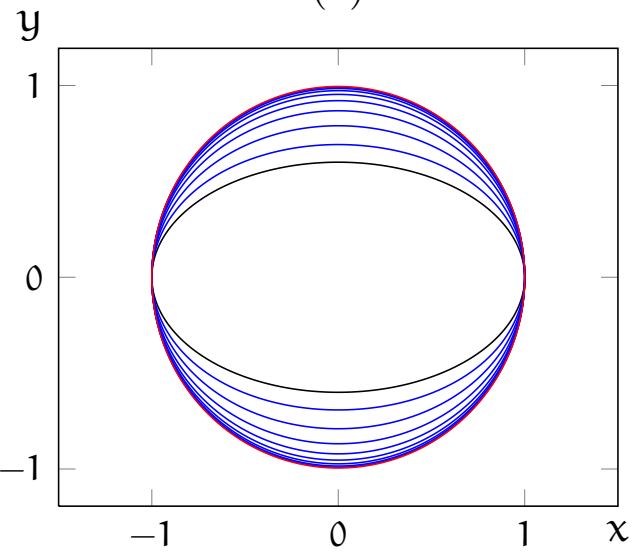

(b)

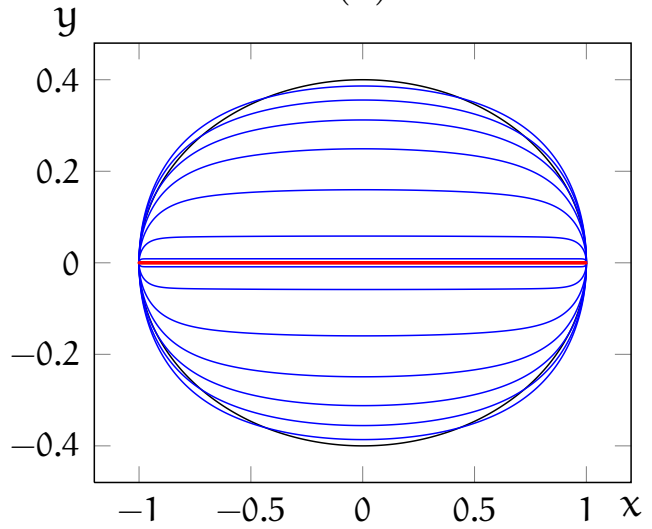

Figure 5: The evolution of initially elliptical shaped bubble (21) using the scaled scheme outlined in Section 2 with $Q=1, \sigma=0.35$ and $c=1$ (initial and final interfaces are black and red, respectively): (a) the aspect ratio is $\beta=0.6$ and the bubble heads to a circle; (b) $\beta=0.4$ and the bubble heads towards an infinitely thin slit, demonstrating the existence of multiple asymptotic bubble shapes for the parameter set $\frac{1}{3}<\sigma /(\mathrm{cQ})<\frac{1}{2}$.

condition of the form

$$
\mathrm{G}(\zeta, 0)=\frac{1+\beta}{2} \frac{1}{\zeta}+\frac{1-\beta}{2} \zeta, \quad 0<\beta<1 .
$$

The parameter $\beta$ is the aspect ratio of the ellipse. In Figure 5 we plot the numerical solution obtained from our scaled method for identical parameter values but slightly different initial conditions. The slightly fatter ellipse tends to a circle, while the thinner tends to an infinitely thin 'slit' as $T \rightarrow \infty$ (the extinction limit). This nonunique extinction behaviour occurs in a specific parameter range $\frac{1}{3}<\sigma /(\mathrm{cQ})<\frac{1}{2}$, a result which we describe in greater depth elsewhere [2]. 


\section{Discussion}

We developed a highly accurate and efficient numerical scheme for computing the evolution of a bubble in Hele-Shaw flow with surface tension and kinetic undercooling effects on the boundary. This scheme is used to demonstrate the effect of the nonlinear boundary conditions on bubbles pinching off into multiple bubbles, and to show the existence of multiple asymptotic bubble shapes, for certain parameter values, for bubbles which shrink to a point.

The scaled version of the scheme is particularly effective at capturing the behaviour of a bubble very close to the point at which it becomes extinct. The method is also capable of demonstrating the onset of instability for an expanding bubble, although it is not as effective at long term finger formation as other boundary integral methods designed for the purpose [12] and level set methods [8], which have the advantage that they continue past pinch off. The numerical results raise opportunities for further work. There is the interesting bifurcation structure in the asymptotic bubble shape which we recently reported [2]. Additionally, we intend to expand upon the phenomenon of pinch off and the effects of the boundary conditions thereon. In particular, we are interested in the existence of non-generic asymptotic bubble shapes that may occur in the limit that pinch off occurs at the theoretical extinction time. This implies the existence of non-elliptical extinction for the unregularised $(\sigma=c=0)$ problem [10], and non-circular extinction for the purely surface tension $(c=0)$ problem.

\section{References}

[1] M. C. Dallaston and S. W. McCue. New exact solutions for Hele-Shaw flow in doubly connected regions. Phys. Fluids, 24:052101, 2012. doi:10.1063/1.4711274. C312 
[2] M. C. Dallaston and S. W. McCue. Bubble extinction in Hele-Shaw flow with surface tension and kinetic undercooling regularisation. Nonlinearity, 26:1639-1665, 2013. doi:10.1088/0951-7715/26/6/1639. C311, C313, C315, C322, C323

[3] E. O. Dias and J. A. Miranda. Control of radial fingering patterns: A weakly nonlinear approach. Phys. Rev. E, 81(1):016312 (1-7), 2010. doi:10.1103/PhysRevE.81.016312. C312

[4] E. O. Dias, E. Alvarez-Lacalle, M. S. Carvalho, and J. S. Miranda. Minimization of viscous fluid fingering: a variational scheme for optimal flow rates. Phys. Rev. Lett., 109:144502, 2012. doi:10.1103/PhysRevLett.109.144502. C312

[5] V. Entov and P. Etingof. On the breakup of air bubbles in a Hele-Shaw cell. Eur. J. Appl. Math., 22:125-149, 2011. doi:10.1017/S095679251000032X. C311, C315

[6] V. M. Entov and P. I. Etingof. Bubble contraction in Hele-Shaw cells. Q. J. Mech. Appl. Math., 44:507-535, 1991. doi:10.1093/qjmam/44.4.507. C311, C315

[7] L. K. Forbes. A cylindrical Rayleigh-Taylor instability: radial outflow from pipes or stars. J. Eng. Math., 70:205-224, 2011. doi:10.1007/s10665-010-9374-z. C312

[8] T. Y. Hou, Z. Li, S. Osher, and H. Zhao. A hybrid method for moving interface problems with application to the Hele-Shaw flow. Journal of Computational Physics, 134(2):236-252, 1997. doi:10.1006/jcph.1997.5689. C310, C323

[9] S. D. Howison. Complex variable methods in Hele-Shaw moving boundary problems. Eur. J. Appl. Math., 3:209-224, 1992. doi:10.1017/S0956792500000802. C311, C312 
[10] J. R. King and S. W. McCue. Quadrature domains and p-Laplacian growth. Complex Anal. Oper. Th., 3:453-469, 2009. doi:10.1007/s11785-008-0103-9. C311, C315, C321, C323

[11] S.-Y. Lee, E. Bettelheim, and P. Weigmann. Bubble break-off in Hele-Shaw flows - singularities and integrable structures. Physica D, 219:22-34, 2006. doi:10.1016/j.physd.2006.05.010. C311

[12] S. Li, J. S. Lowengrub, and P. H. Leo. A rescaling scheme with application to the long-time simulation of viscous fingering in a Hele-Shaw cell. J. Comp. Phys., 225(1):554-567, 2007. doi:10.1016/j.jcp.2006.12.023. C312, C323

[13] S. Li, J. S. Lowengrub, J. Fontana, and P. Palffy-Muhoray. Control of viscous fingering patterns in a radial Hele-Shaw cell. Phys. Rev. Lett., 102(17):174501, 2009. doi:10.1103/PhysRevLett.102.174501. C312

[14] S. W. McCue and J. R. King. Contracting bubbles in Hele-Shaw cells with a power-law fluid. Nonlinearity, 24:613-641, 2011. doi:10.1088/0951-7715/24/2/009. C311

[15] S. W. McCue, J. R. King, and D. S. Riley. Extinction behaviour of contracting bubbles in porous media. Q. J. Mech. Appl. Math., 56:455-482, 2003. doi:10.1093/qjmam/56.3.455. C315

[16] S. W. McCue, J. R. King, and D. S. Riley. Extinction behavior for two-dimensional inward-solidification problems. Proc. R. Soc. Lond. A, 459:977-999, 2003. doi:10.1098/rspa.2002.1059. C310

[17] S. W. McCue, J. W. King, and D. S. Riley. The extinction problem for three-dimensional inward solidification. J. Eng. Math., 52:389-409, 2005. doi:10.1007/s10665-005-3501-2. C310

[18] M. Reissig, D. V. Rogosin, and F. Hübner. Analytical and numerical treatment of a complex model for Hele-Shaw moving boundary value problems with kinetic undercooling regularization. Eur. J. Appl. Math., 10:561-579, 1999. http: 
//journals. cambridge. org/action/displayAbstract?fromPage= online\&aid=44021\&fulltextType=RA\&f ile Id=S0956792599003939 C311

[19] P. G. Saffman and G. I. Taylor. The penetration of a fluid into a porous medium or Hele-Shaw cell containing a more viscous liquid. Proc. $R$. Soc. Lond. A, 245:312-329, 1958. doi:10.1098/rspa.1958.0085. C310, C312

\section{Author addresses}

1. Michael C. Dallaston, School of Mathematical Sciences, Queensland Univerisity of Technology, Queensland 4000, Australia. mailto:michael.dallaston@qut.edu .au

2. Scott W. McCue, School of Mathematical Sciences, Queensland Univerisity of Technology, Queensland 4000, Australia. mailto:scott.mccue@qut.edu.au 